\title{
Electron Spin Resonance in sine-Gordon spin chains in the perturbative spinon regime
}

\author{
S. A. Zvyagin, ${ }^{1}$ A. K. Kolezhuk, ${ }^{2, *}$ J. Krzystek, ${ }^{3}$ and R. Feyerherm ${ }^{4}$ \\ ${ }^{1}$ Hochfeld-Magnetlabor Dresden, Forschungszentrum Rossendorf, 01328 Dresden, Germany \\ ${ }^{2}$ Institut für Theoretische Physik, Universität Hannover, 30167 Hannover, Germany \\ ${ }^{3}$ National High Magnetic Field Laboratory, Tallahassee, FL 32310 \\ ${ }^{4}$ Hahn-Meitner-Institut (HMI), 14109 Berlin, Germany
}

\begin{abstract}
We report the low-temperature multi-frequency ESR studies of copper pyrimidine dinitrate, a spin- $\frac{1}{2}$ antiferromagnetic chain with alternating $g$-tensor and the Dzyaloshinskii-Moriya interaction, allowing us to test a new theoretical concept proposed recently by Oshikawa and Affleck [Phys. Rev. Lett. 82, 5136 (1999)]. Their theory, based on bosonization and the self-energy formalism, can be applied for precise calculation of ESR parameters of $S=\frac{1}{2}$ antiferromagnetic chains in the perturbative spinon regime. Excellent quantitative agreement between the theoretical predictions and experiment is obtained.
\end{abstract}

PACS numbers: 75.40.Gb, 76.30.-v, 75.10.Jm

Quantum fluctuations in low-dimensional magnets give rise to a variety of exotic strongly correlated states, making those systems an extremely attractive ground for testing various theoretical concepts. Isotropic $S=\frac{1}{2}$ Heisenberg antiferromagnetic (AFM) chains with uniform nearest-neighbor exchange interactions have a spin singlet ground state; their spin dynamics is determined by a gapless two-particle continuum of spin- $\frac{1}{2}$ excitations (spinons), whose parameters can be calculated using Bethe ansatz [1]. In an applied magnetic field $H$ the excitation spectrum acquires incommensurate modes but remains gapless [2, 3]. In contrast, in $S=\frac{1}{2}$ AFM chains with an alternating $g$-tensor and the DzyaloshinskiiMoriya (DM) interaction, a magnetic field induces an energy gap $E_{g} \sim H^{2 / 3}$ [4], with the effective transverse staggered field [4, 5] playing a key role in the gap formation. Fairly complete theoretical understanding of this phenomenon has been achieved by Oshikawa and Affleck [5, 6] who showed that the gapped phase could be effectively described using the sine-Gordon quantum field theory. A rich excitation spectrum [7] was predicted to be readily apparent in the response functions 81. Recently, with the help of inelastic neutron scattering 9| and electron spin resonance (ESR) techniques 10, 11, 12] the existence of soliton and breather states in sine-Gordon spin chains has been confirmed experimentally.

ESR is traditionally recognized as one of the most powerful and extremely sensitive tools for probing the magnetic excitation spectrum in exchange-coupled spin systems. Using the temperature or magnetic field as a tuning parameter, one can obtain valuable information on the nature of the ground state and estimate their important physical parameters and constants. The lowdimensional (low-D) spin systems are of particular interest for ESR (see, for instance, Ref. 13, 14] and references therein). However, lack of a proper ESR theory often made a detailed interpretation of experimental data, and thus an accurate comparison with proposed models rather problematic. A new theoretical approach for cal- culating ESR parameters of $S=\frac{1}{2}$ AFM chains, which is based on bosonization and the standard Feynman-Dyson self-energy formalism, has been recently developed by Oshikawa and Affleck 15. Importantly, the new concept avoids the Hartree-Fock approximation, which was previously used [16] for interpreting ESR in exchangecoupled spin systems, but appears to be generally invalid in $1 \mathrm{D}$ magnets.

In a general case the Hamiltonian of a spin- $\frac{1}{2}$ Heisenberg AFM chain in an external field is given by

$$
\mathcal{H}=J \sum_{i} \vec{S}_{i} \cdot \vec{S}_{i+1}+g \mu_{B} H \sum_{i} S_{i}^{z}+\mathcal{H}_{\delta},
$$

where the first term corresponds to the isotropic Heisenberg interaction, the second one is the Zeeman term and the third one represents various possible small anisotropic contributions. In sine-Gordon spin chains the term $\mathcal{H}_{\delta}$ in (11) is dominated by the effective staggered field $h=c H[4,[5]$ originating from the alternating $g$-tensor and the DM interaction, $\mathcal{H}_{\delta}=g \mu_{B} h \sum_{i}(-1)^{i} S_{i}^{x}$. If the temperature is high enough to destroy the solitonbreather superstructure, but sufficiently small compared to the characteristic energy of the exchange interaction $J$, the term $\mathcal{H}_{\delta}$ can be regarded as a perturbation. In its absence, i.e., in an ideal $S=\frac{1}{2}$ Heisenberg AFM chain, the resonance would occur exactly at the frequency $\hbar \omega=g \mu_{B} H$ and have a zero linewidth in the low-temperature limit, due to the conservation of the total $S^{z}$. In the Oshikawa-Affleck (OA) theory 15, the sole effect of the symmetry-breaking perturbation $\mathcal{H}_{\delta}$ on the Green function is to produce the self-energy contribution $\Sigma$ whose real and imaginary part determine the frequency shift and the linewidth, respectively. Other possible symmetry-breaking contributions arising, e.g., from the exchange anisotropy, can be distinguished by the characteristic dependence of the linewidth and the frequency shift on the magnetic field $H$ and the temperature $T$ [15]. Importantly, the OA theory allows a precise calculation of the ESR parameters and their dependence 
on temperature and magnetic field. Once proven, this approach can be used for studying peculiarities of magnetic microstructure of $S=\frac{1}{2}$ AFM chains, and investigating anisotropies of magnetic interactions, including those originating from the staggered field effects.

For the first time the significant changes in ESR spectra at low temperatures were observed in the spin- $\frac{1}{2}$ antiferromagnetic chain system with alternating $g$-tensor and the DM interaction copper benzoate 17, 18] (although the data were mistakenly explained in terms of the $3 \mathrm{D}$ ordering scenario). Later on, it was found [10] that terms $(H / T)^{2}$ and $(H / T)^{3}$ clearly dominated in the ESR linewidth and shift of the resonance field, respectively, being consistent with the OA predictions. Bertaina et al. [19] have recently studied the ESR linewidth behavior in the quasi-1D antiferromagnet $\mathrm{BaCu}_{2} \mathrm{Ge}_{2} \mathrm{O}_{7}$. It appeared that an enhancement of $3 \mathrm{D}$ long-range correlations in $\mathrm{BaCu}_{2} \mathrm{Ge}_{2} \mathrm{O}_{7}$ in the vicinity of the AFM transition at $T_{N}=8.8 \mathrm{~K}$ significantly affected the low temperature ESR behavior, which made an accurate quantitative comparison with the theory difficult [20]. Thus one may conclude that at a quantitative level a convincing evidence for the validity of the low-temperature ESR theory for $S=\frac{1}{2}$ AFM chains with alternating $g$-tensor and the Dzyaloshinskii-Moriya interaction is still lacking.

In this Letter we report a detailed ESR study of copper pyrimidine dinitrate $\left(\left[\mathrm{PM}-\mathrm{Cu}\left(\mathrm{NO}_{3}\right)_{2}\left(\mathrm{H}_{2} \mathrm{O}\right)_{2}\right]_{n}\right.$, PM = pyrimidine; hereafter $\mathrm{Cu}-\mathrm{PM})$, a spin- $\frac{1}{2}$ antiferromagnetic chain with alternating $g$-tensor and the DM interaction, which appears to be the most perfect realization of the quantum sine-Gordon spin chain system known to date. The measurements were performed in the intermediate ("perturbative spinon") temperature regime, $E_{g}<T<J$, at several frequencies, allowing us to check both the temperature and field dependencies of ESR parameters, predicted by Oshikawa and Affleck [15. The obtained data were of a sufficient quality to perform an accurate quantitative comparison with theoretical predictions, achieving an excellent agreement between the theory and experiment. Importantly, our results are fully consistent with the previous analysis 12 based on the study of the frequency-field dependencies of ESR excitations in $\mathrm{Cu}-\mathrm{PM}$ in the soliton-breather regime.

$\mathrm{Cu}-\mathrm{PM}$ crystallizes in a monoclinic structure belonging to the space group $C 2 / c$ with four formula units per unit cell 21]. The lattice constants obtained from the singlecrystal X-ray diffraction are $a=12.404 \AA, b=11.511 \AA$, $c=7.518 \AA, \beta=115.0^{\circ}$. The $\mathrm{Cu}$ ions form chains running parallel to the short $a c$ diagonal. The $\mathrm{Cu}$ coordination is a distorted octahedron, built from an almost square $\mathrm{N}-\mathrm{O}-\mathrm{N}-\mathrm{O}$ equatorial plane and two oxygens in the axial positions. In this approximately tetragonal local symmetry, the local principal axis of each octahedron is tilted from the $a c$ plane by $\pm 29.4^{\circ}$. Since this axis almost coincides with the principal axis of the $g$ tensor, the $g$-tensors for neighboring $\mathrm{Cu}$ ions are stag-

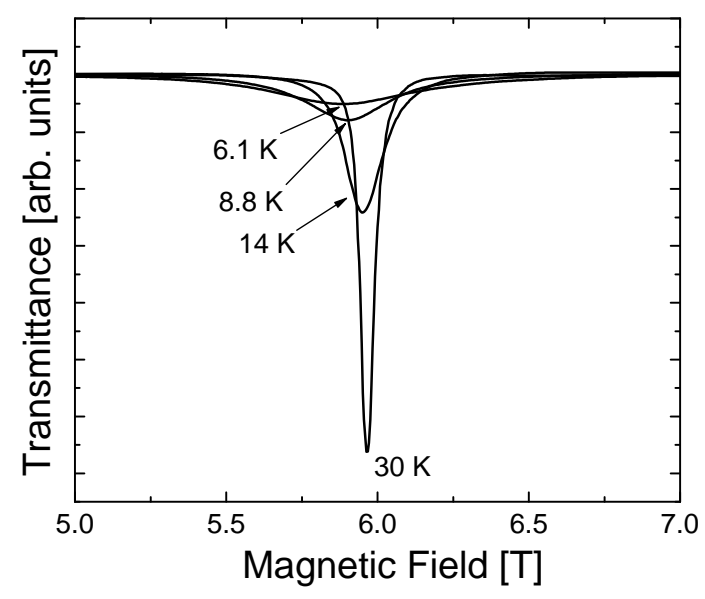

FIG. 1: The ESR transmission spectra of Cu-PM taken at a frequency of $184 \mathrm{GHz}$ at four different temperatures.

gered. Magnetic properties of $\mathrm{Cu}-\mathrm{PM}$ are well characterized 12, 21, 22, 23, 24]. The exchange spin interaction $J$ was found to be $36 \mathrm{~K}$ [21], while the staggered field parameter, $c$, was estimated as 0.08 [12]. Recently, the excitation spectrum of $\mathrm{Cu}-\mathrm{PM}$ in the soliton-breather regime $\left(T \ll E_{g}\right)$ has been studied in detail [12], using the tunable-frequency ESR technique 25]. Signatures of soliton and three breather branches have been clearly identified, and the field-induced gap has been observed directly. In our experiments high quality single crystals were used, with the magnetic field applied along the $c^{\prime \prime}$ direction providing a maximum staggered field 21, 22]. The ESR absorptions were fit using the Lorentzian formula for the line shape. Usually, ESR probes the imaginary part of dynamical susceptibility, $\chi(q, \omega)$, responsible for the absorption. However, even a small admixture of the real component of $\chi(q, \omega)$ (responsible for the dispersion) can significantly distort the resonance lineshape and thus can be an additional source of experimental error. To minimize the contribution of the dispersive component in the ESR spectra, special steps associated with a careful control and correction of the microwave phase were made. An accuracy of better than $5 \%$ and $0.2 \%$ was achieved for calculating ESR linewidth and $g$-factor, respectively.

Typical absorption spectra in $\mathrm{Cu}-\mathrm{PM}$ at frequency of $184 \mathrm{GHz}$ at four different temperatures are shown in Fig. [1. One can see that upon cooling the high-temperature $\omega=g H$ resonance becomes broader, while the absorption maximum shifts towards lower fields. Such a behavior is consistent with that observed earlier in copper benzoate [10] and $\mathrm{BaCu}_{2} \mathrm{Ge}_{2} \mathrm{O}_{7}[19]$. In our experiments the temperature dependence of the linewidth has been studied at three frequencies, 9.4, 93.1, and $184 \mathrm{GHz}$. The corresponding data are summarized in Fig. 22 together with results of the OA theory. To fit the data, the following 


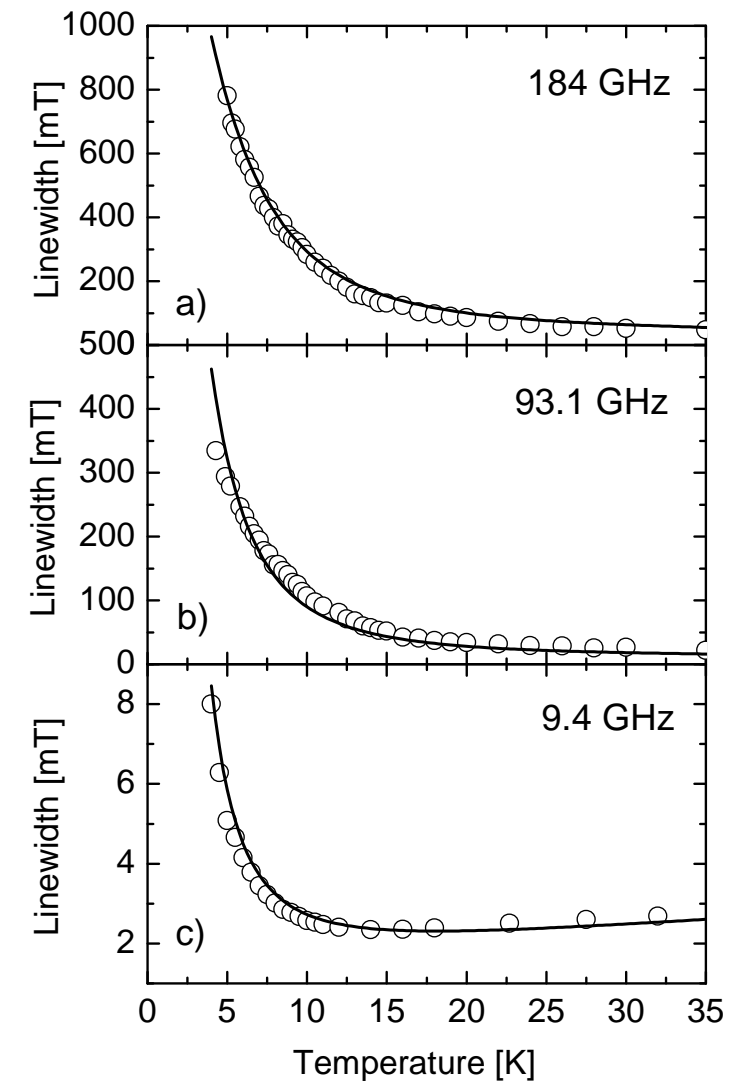

FIG. 2: The temperature dependence of the ESR linewidth in $\mathrm{Cu}-\mathrm{PM}$ at frequencies of 184, 93.1, and 9.4 GHz. Symbols denote the experimental results, and solid lines correspond to the best global fit using Eq. (2).

expression 15] has been used for the ESR linewidth $\Delta H$ :

$$
\Delta H=\eta_{0}+F z H \operatorname{Im}(G),
$$

where $z=\Gamma\left(\frac{1}{4}\right) / \Gamma\left(\frac{3}{4}\right)$ and $\Gamma(x)$ denotes the gamma function, and

$$
\begin{aligned}
& G(H, T)=\Gamma\left(\frac{1}{4}-i \frac{g \mu_{B} H}{2 \pi T}\right) / \Gamma\left(\frac{3}{4}-i \frac{g \mu_{B} H}{2 \pi T}\right), \\
& F(H, T)=c^{2} \sqrt{\pi / 128}(J / T) \ln ^{1 / 2}(\lambda J / T) .
\end{aligned}
$$

The constant $\lambda$ in the leading $\log$ is to be viewed as a free phenomenological parameter compensating for the effects of subleading logarithmic terms [26]. Subleading logs were recently exactly determined for an exchange anisotropy perturbation [28], but for the staggered field case their exact form is not known. The overall correction $\eta_{0}$ takes into account that the ESR linewidth is in fact finite in the high-temperature $(T \gtrsim J)$ regime: According to the OA theory, for $T \gg J$ the linewidth should become temperature-independent,

$$
\Delta H \mapsto \eta_{0}=\alpha+\beta\left(g \mu_{B} H / J\right)^{2},
$$

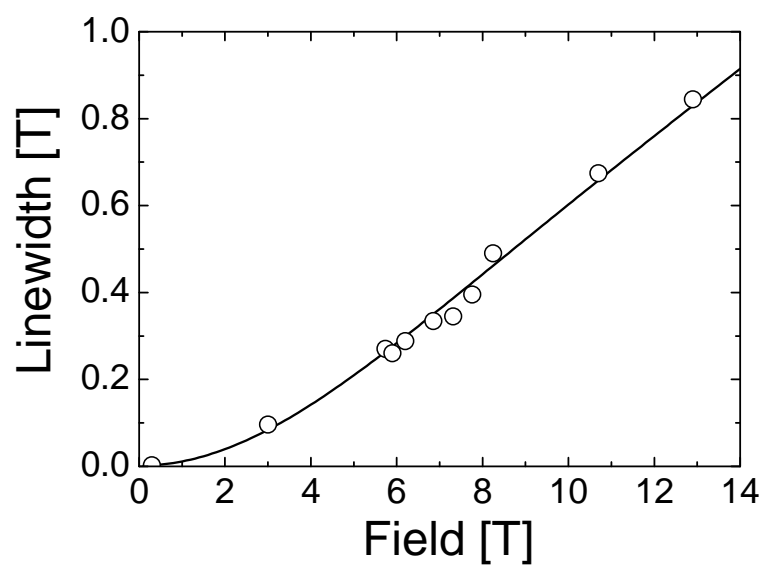

FIG. 3: The field dependence of the ESR linewidth in $\mathrm{Cu}-\mathrm{PM}$ at $T=10.3 \mathrm{~K}$. The solid line corresponds to Eq. (2) with the parameters obtained from the fit as described in the text.

finite $\alpha$ being caused by the DM interaction, and $\beta$ containing contributions both from the DM interaction and from staggered $g$-tensor [15].

We obtain a very good fit to the entire set of the linewidth data, as shown in Fig. 2] with the following values of the parameters: $c=0.083 \pm 0.001, \lambda=2.0 \pm 0.05$, $\alpha=(1.5 \pm 0.2) \mathrm{mT}$, and $\beta=(830 \pm 25) \mathrm{mT}$. It should be remarked that inclusion of log corrections turns out to be important: neglecting them makes it impossible to achieve a uniformly good fit for different frequencies. The obtained staggered field parameter $c$ is in excellent agreement with the value $c=0.08 \pm 0.002$ found by us earlier from the analysis of the frequency-field dependence of ESR modes in $\mathrm{Cu}-\mathrm{PM}$ in the soliton-breather regime [12. The field dependence of the linewidth measured in fields up to $13 \mathrm{~T}$ at temperature of $10.3 \mathrm{~K}$ is shown in Fig. [3 together with the results of calculations using parameters obtained as described above. The data are in excellent agreement with the calculated values.

As seen in Fig. 2( c), at $9.4 \mathrm{GHz}$ the temperature dependence of the linewidth contains a tiny (note the scale) contribution linearly growing in $T$. According to OA, this can be caused by a small exchange anisotropy $\delta$, yielding the linewidth contribution $\left(4 / \pi^{3}\right)(\delta / J)^{2} T$ if one assumes the anisotropy axis to be parallel to the applied field [15]. Our data at $9.4 \mathrm{GHz}$ allow us to estimate this anisotropy $(\delta / J)$ as being around $1.2 \%$ which is consistent with the susceptibility data estimating it to be within $2 \%$ [21].

Let us now discuss the behavior of $g$-factor. It is worth to mention here that while the linewidth in $\mathrm{Cu}-\mathrm{PM}$ can be relatively easily measured, an accuracy of measurements becomes really an important problem when measuring the $g$-factor dependence, particularly at low frequency. Employing the high-frequency and -field ESR techniques enabled us to achieve much better resolution 


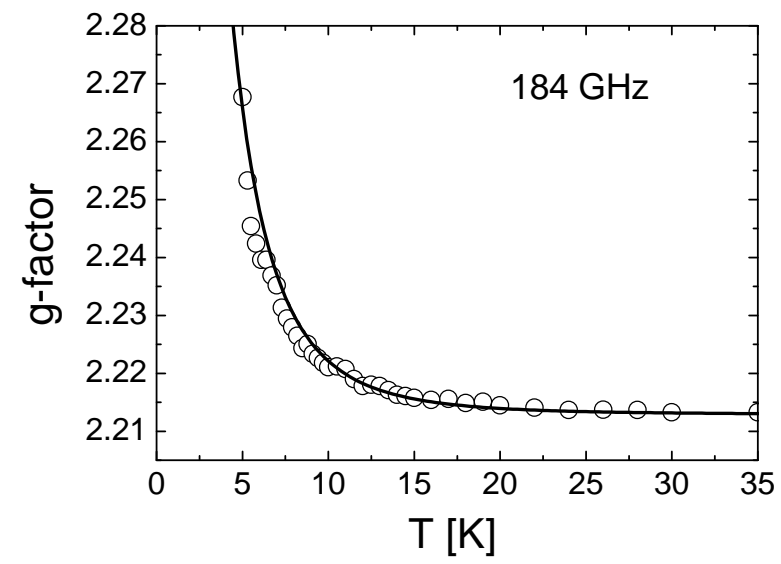

FIG. 4: The temperature dependence of the effective $g$-factor in $\mathrm{Cu}-\mathrm{PM}$ for a frequency of $184 \mathrm{GHz}$. The solid line corresponds to Eq. (4) with the parameters obtained from the linewidth fit as described in the text.

than conventional low-frequency methods 22], and thus to significantly improve the accuracy of measurements. The experimental data for the dimensionless resonance frequency shift $\Delta g$ (the $g$-factor shift with respect to the $g$ value at room temperature, $g=2.215$ ) for a frequency of $184 \mathrm{GHz}$ are presented in Fig. 4 together with the theoretical curve described by the expression 15 .

$$
\Delta g=F z(z-\operatorname{Re}(G)) .
$$

The same parameter values as obtained from fitting the linewidth data has been used. An excellent agreement with the theory can be seen. For the chosen value of $H$ the breather gap $E_{g} \approx 6 \mathrm{~K}$, which shows that the OA theory actually works fairly well down to $T \sim E_{g}$.

In summary, we have presented a detailed study of the temperature and field evolution of the ESR spectrum in $\mathrm{Cu}-\mathrm{PM}$, a material which is considered as the best available realization of spin- $\frac{1}{2}$ Heisenberg chain with alternating $g$-tensor and the Dzyaloshinskii-Moriya interaction, in the perturbative spinon regime. The data were analyzed in frame of the Oshikawa-Affleck quantum-field theory 15. An excellent quantitative agreement with the theory has been achieved. The results are fully consistent with the previous analysis 12 based on the study of the ESR excitation spectrum of $\mathrm{Cu}-\mathrm{PM}$ in the solitonbreather regime.

Acknowledgments.- The authors express their sincere thanks to C.L. Broholm, F.H.L. Essler, A. Honecker, H.H. Klauss, J.L. Musfeldt, M. Oshikawa, P. Schlottmann, G. Tetel'baum and A.U.B. Wolter for fruitful discussions. We would like to thank P.J. Desrochers for critical reading of the manuscript and useful comments. The experimental part of this work was supported by NSF Cooperative Agreement No. DMR-0084173 and by the State of Florida. AK is supported by the Heisenberg Fellowship of Deutsche Forschungsgemeinschaft.
* On leave from: Institute of Magnetism, National Academy of Sciences and Ministry of Education, 03142 Kiev, Ukraine.

[1] L. D. Faddeev and L. A. Takhtajan: Phys. Lett. 85A, 375 (1981)

[2] G. Müller et al., Phys. Rev. B 24, 1429 (1981).

[3] M. B. Stone et al., Phys. Rev. Lett. 91, 037205 (2003).

[4] D. C. Dender et al., Phys. Rev. Lett. 79, 1750 (1997).

[5] M. Oshikawa and I. Affleck, Phys. Rev. Lett. 79, 2883 (1997).

[6] I. Affleck and M. Oshikawa, Phys. Rev. B 60, 1038 (1999); ibid. 62, 9200 (2000).

[7] R. Dashen et al., Phys. Rev. D 11, 3424 (1975).

[8] F. H. L. Essler and A. M. Tsvelik, Phys. Rev. B 57, 10592 (1998); F. H. L. Essler et al., Phys. Rev. B 68, 064410 (2003).

[9] M. Kenzelmann et al., Phys. Rev. Lett. 93, 017204 (2004).

[10] T. Asano et al., Phys. Rev. Lett. 84, 5880 (2000).

[11] T. Asano et al., Physica B 329, 1213 (2003).

[12] S. A. Zvyagin et al., Phys. Rev. Lett. 93, 027201 (2004).

[13] K. Katsumata, J. Phys. Cond. Matter 12, R589 (2000).

[14] S. A. Zvyagin, in Spectroscopy of Emerging Materials, ed. by E.C. Faulques et al. (Kluwer Academic Publishers, Netherland, 2004).

[15] M. Oshikawa and I. Affleck, Phys. Rev. Lett. 82, 5136 (1999); Phys. Rev. B 65, 134410 (2002).

[16] H. Mori and K. Kawasaki, Prog. Theor. Phys. 27, 529 (1962); 28, 971 (1962).

[17] M. Date et al., Prog. Theor. Phys. Suppl. 46, 194 (1970).

[18] K. Okuda et al., J. Phys. Soc. Jpn. 33, 1574 (1972).

[19] S. Bertaina et al., Phys. Rev. Lett. 92, 057203 (2004).

[20] Not underestimating the importance of the work of Bertaina et al. [19], we have to mentnion here that there is a definite deviation of the calculated values from the experimental results for the low-temperature part of the linewidth temperature dependencies measured at frequencies of $34 \mathrm{GHz}$ and $70.6 \mathrm{GHz}$ (see Fig. 2b of Ref. [19]). A substantial inconsistency in the extracted values of the parameter $c$ has been observed: it was found that the OA calculations fit relatively well to the ESR data giving $c=0.09$, which is about $35 \%$ less than the value $c=0.14$ found from the analysis of the frequency-field dependence of magnetic excitations in the AFM longrange ordered phase. Finally, the subtraction of the 3D contribution undertaken in Ref. [19] requires additional assumptions not contained in the OA theory.

[21] R. Feyerherm et al., J. Phys.: Condens. Matter 12, 8495 (2000).

[22] T. Asano et al., Physica B 329, 1006 (2003).

[23] A. U. B. Wolter et al., Phys. Rev. B 68, 220406(R) (2003).

[24] A. U. B. Wolter et al., Phys. Rev. Lett. 94, 057204 (2005).

[25] S. A. Zvyagin et al., Physica B 346, 1 (2004).

[26] Note that the correct power of the leading log term is $\frac{1}{2}$ and not 1 as in Ref. 15] (formulas presented in Ref. 15] contain a misprint [27]).

[27] M. Oshikawa, private communication.

[28] Y. Maeda, K. Sakai, and M. Oshikawa, preprint arXiv:cond-mat/0501295 